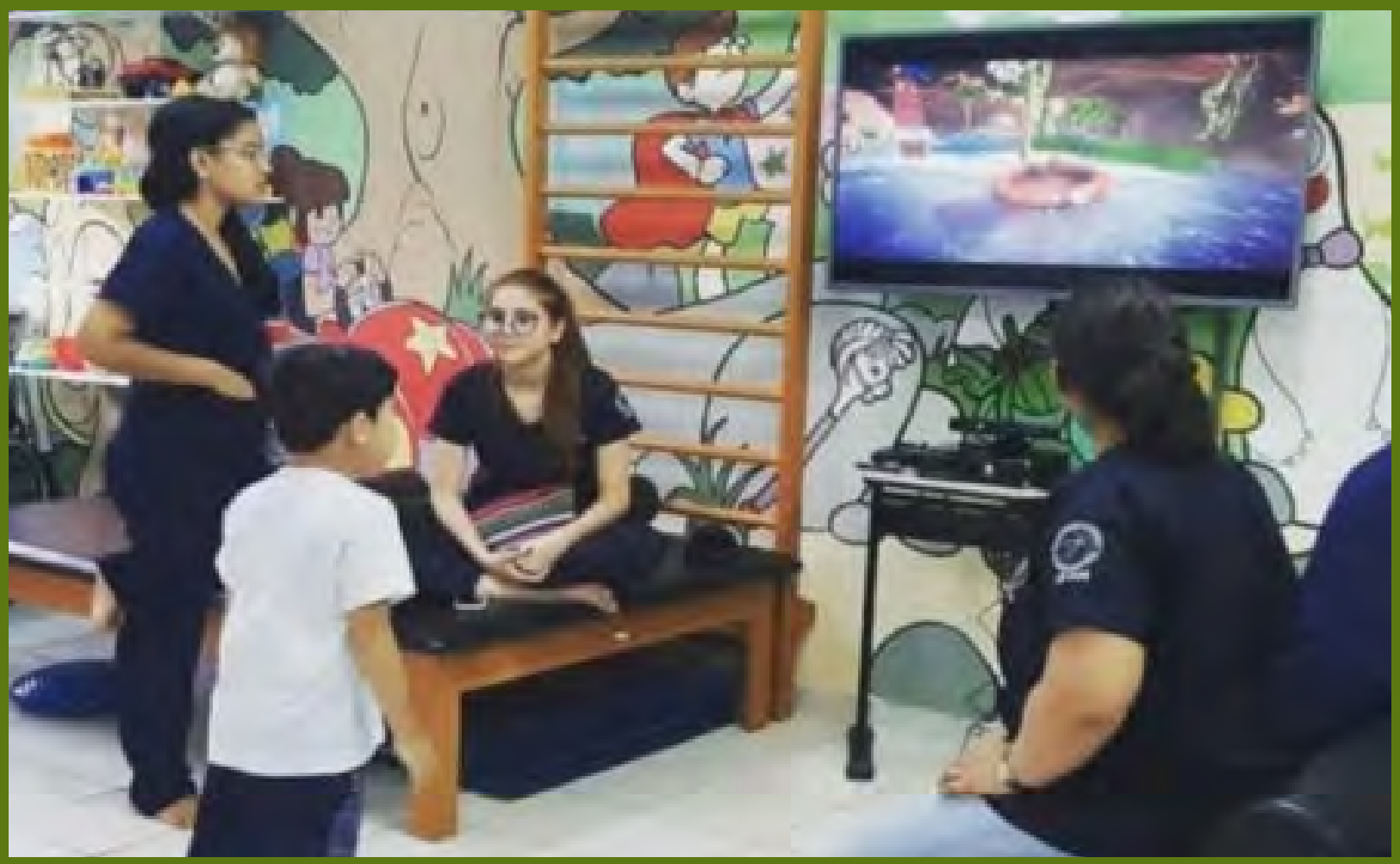

\title{
PROPED - PROGRAMA DE REABILITAÇÃO PEDIÁTRICA: relato de experiência de projeto extensionista
}

\author{
Lohanna Pinheiro Martins - lohanna_martins@hotmail.com ${ }^{1}$ \\ Michelle Alexandrina dos Santos Furtado - alexandrinamichelle@gmail.com ${ }^{2}$ \\ Karoliny Lisandra Teixeira Cruz - karol.lisandra@outlook.com ${ }^{3}$ \\ Ayrles Silva Gonçalves Barbosa Mendonça - ayrles@yahoo.com.br ${ }^{4}$
}

\section{RESUMO}

0 trabalho do fisioterapeuta pediátrico consiste em proporcionar promoção à saúde infantil, de maneira holística, lúdica e de forma individualizada. 0 objetivo do trabalho é relatar a vivência dos atendimentos realizados no Programa de Reabilitação Pediátrica (PROPED) da Universidade Federal do Amazonas (UFAM), por meio das ações de seus membros e colaboradores. A reabilitação é voltada à população pediátrica através dos atendimentos e da assistência aos seus respectivos pais e cuidadores. O PROPED faz parte do Programa de Atividade Curricular de Extensão (PACE) da UFAM e suas ações acontecem semestralmente desde 2013, sendo o presente relato dedicado a descrever as atividades realizadas ao longo de sete anos de permanente atuação (2013 a 2019). Ao todo compuseram o projeto 20 colaboradores e mais de 60 alunos estiveram envolvidos nas atividades e funcionamento do programa. Atividades envolvidas com ensino e pesquisa também fizeram parte do repertório do PROPED, incluindo apresentação de trabalhos em eventos científicos, elaboração de casos clínicos, capacitação dos discentes em escalas de avaliação, métodos de intervenção e fabricação de órteses termomoldáveis.

1 Fisioterapeuta colaboradora do PROPED/UFAM.

2 Fisioterapeuta, Mestranda em Reabilitação e Desempenho Funcional pela Universidade Federal dos Vales de Jequitinhonha e Mucuri - UFVJM.

3 Fisioterapeuta, Mestra em Reabilitação e Desempenho Funcional pela Universidade Federal dos Vales de Jequitinhonha e Mucuri - UFVJM.

4 Fisioterapeuta, Professora Doutora Adjunta do curso de Fisioterapia da Universidade Federal do Amazonas - UFAM, coordenadora do PROPED. 


\title{
THE PEDIATRIC REHABILITATION PROGRAM (PROPED): the Extension Program experience report
}

\begin{abstract}
The pediatric physiotherapist's job is to promote child health in a holistic, playful and individualized way. The objective of the work is to report the experience of care provided in the Pediatric Rehabilitation Program (PROPED) of the Federal University of Amazonas (UFAM), through the actions of its members and collaborators. The rehabilitation is aimed at the pediatric population through the attendance and assistance to their respective parents and caregivers. PROPED is part of the Curricular Extension Program of UFAM (PACE) and its actions have taken place every six months since 2013 , and the present report describes the activities carried out over seven years of permanent activity (from 2013 to 2019). In total, 20 employees made up the project and more than 60 students were involved in the activities and operation of the program. Activities that involved teaching and research were also part of PROPED's repertoire, including presentations of works at scientific events, the preparation of clinical cases, the training of students in assessment scales, intervention methods and the manufacture of thermoformable orthoses.
\end{abstract}

KEYWORDS: child development; physiotherapy; pediatrics; re(ha)bilitation.

\section{RELATO DE EXPERIÊNCIA}

0 desenvolvimento motor (DM) infantil é um processo progressivo e contínuo, que ocorre mediante a maturação do sistema nervoso central e interação com sistemas orgânicos e externos, permitindo a aquisição de marcos e habilidades motoras que irão proporcionar adaptações gradativas da criança às constantes mudanças do meio em que está inserida (PEREIRA et al, 2016).

Crianças com alterações neurológicas ou que apresentem DM atípico merecem especial atenção e ações específicas, já que os problemas de coordenação, equilíbrio, postura e controle do movimento poderão se prolongar atéa fase adulta (PEREIRA; VALENTINI; SACCANI, 2016). Dessa forma, o trabalho do fisioterapeuta pediátrico consiste em proporcionar um tratamento holístico e lúdico, visando meios que integrem avaliação, planejamento e intervenções individualizados, além das reavaliações, orientações e educação em saúde que integram o programa terapêutico (BATAGLION; MARINHO, 2016; SILVA; VALENCIANO; FUJISAWA, 2017).

Os projetos de extensão universitários que realizam reabilitação como atividade extensionista tem se tornado uma opção terapêutica para aquelas crianças que não conseguem atendimento na rede pública de saúde, além de oportunizar uma prática não-prescritora aos discentes, fazendo com que os mesmos tenham a experiência através da aplicação prática dos conhecimentos ensinados na academia (BAENA, 2011).

Portanto, o objetivo deste trabalho é relatar a vivência e importância dos atendimentos realizados pelo PROPED no âmbito dos colaboradores do projeto vinculados ao curso de Fisioterapia da Faculdade de Educação Física e Fisioterapia (FEFF), da Universidade Federal do Amazonas (UFAM).

O PROPED faz parte do Programa de Atividade Curricular de Extensão (PACE) da UFAM. Essa modalidade de ação acompanha as atividades do semestre letivo da instituição, sendo composta por um coordenador, vice-coordenador, colaboradores e discentes dos cursos de fisioterapia e engenharia de materiais da UFAM. Sua trajetória se iniciou em 2013, no campo de atuação do ambulatório de Fisioterapia da Fundação Hospital Adriano Jorge (FHAJ), onde permaneceu com suas atividades por três anos. Em 2016, o programa migrou para a Associação dos Pais e Amigos dos Excepcionais (APAE-AM), vinculado ao estágio curricular do curso de Fisioterapia. Desde 2018, os atendimentos vêm sendo realizados na UFAM, no Laboratório de Pediatria da Faculdade de Educação Física e Fisioterapia - FEFF (Figura 1). Tal migração permitiu estreitar o diálogo e as ações com pesquisadores/professores e discentes do curso de engenharia de materiais, o que proporcionou a manufatura de órteses simples com polímeros termomoldáveis (termoplásticos de baixa temperatura), direcionadas principalmente para correção de desvio ulnar e flexão de punhos e dedos, presente comumente nos casos de paralisia cerebral espástica. 


\section{Figura 1 - Vista Geral do Laboratório de Pediatria da Faculdade de Educação Física e Fisioterapia - FEFF}

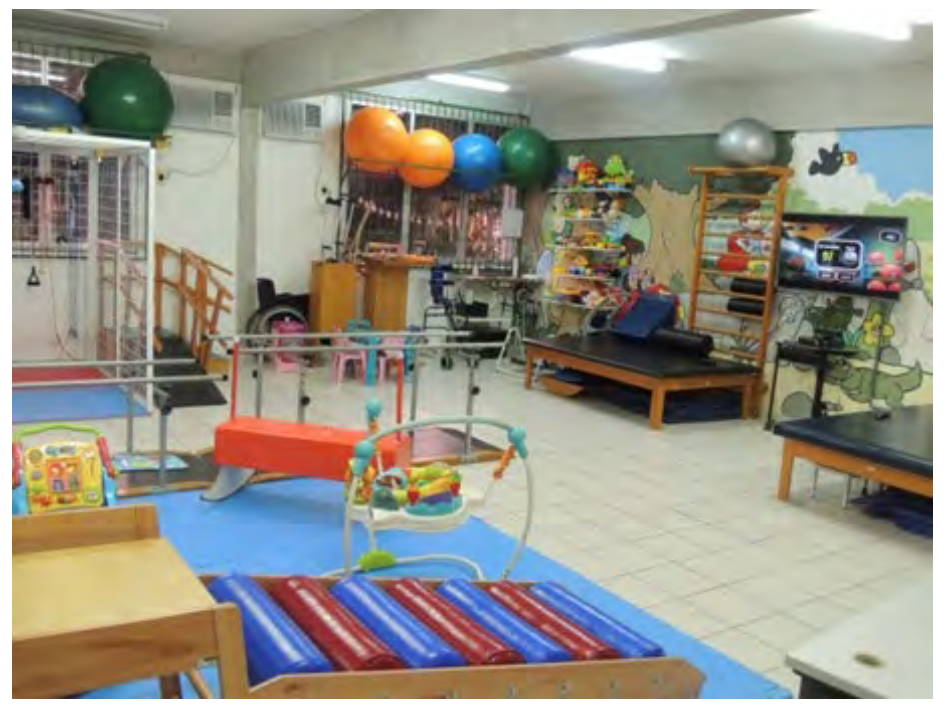

FONTE: Imagem original dos autores.

Ressalta-se que a comunicação entre os cursos e consequente atuação interdisciplinar permitiu a abertura de diálogos cada vez mais estreitos entre os saberes de diferentes áreas do conhecimento, o que proporcionou experiências valiosas tanto para os discentes (de fisioterapia e engenharia) quanto para os profissionais envolvidos, já que a troca de conhecimentos gerou ampliação sobre as áreas de atuação e proporcionou ações multidisciplinares. Assim, ao estudar os casos em conjunto e como uma determinada órtese (por exemplo: manufaturada com materiais de baixo custo e processo de fabricação simples) poderia influenciar no cotidiano e reabilitação dos pacientes, houve um aprofundamento teórico-metodológico das ações de ensino, pesquisa e extensão, o que culminou no desenvolvimento de um novo projeto de extensão, na modalidade PACE, intitulado "Oficina de Ortetização", sob coordenação de um dos colaboradores do PROPED e professor do curso de engenharia de materiais.

A adaptação do PROPED ao laboratório proporcionou um campo de atuação com maiores recursos/ infraestrutura aos atendimentos, tendo em vista que possui áreas específicas (estações) para atuação em diferentes métodos/técnicas de intervenção, como: gameterapia, cinesioterapia, terapia auxiliada por gaiola de estimulação, estimulação à marcha e confecção de pequenas órteses; caracterizando um espaço pensado e adaptado à população infantil, com aproximadamente $100 \mathrm{~m}^{2}$ (Figura 2 e 3).

\section{Figura 2 - Espaço interno do laboratório destinado à Gameterapia/Realidade Virtual}

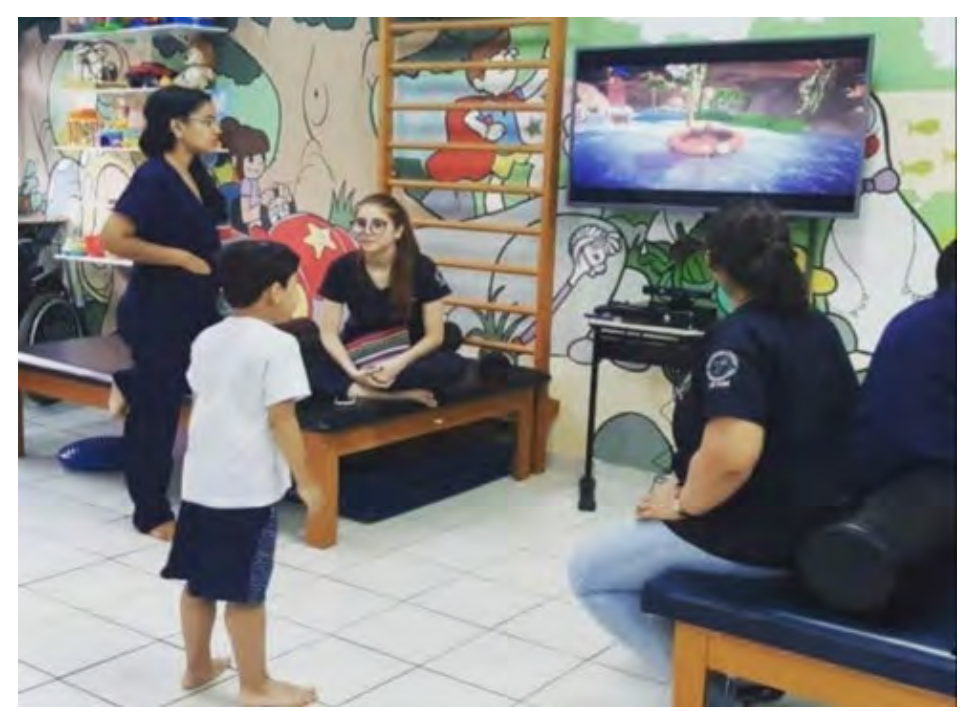

FONTE: Imagem original dos autores. 
Figura 3 - Gaiola para estimulações baseada no conceito Pediasuit.

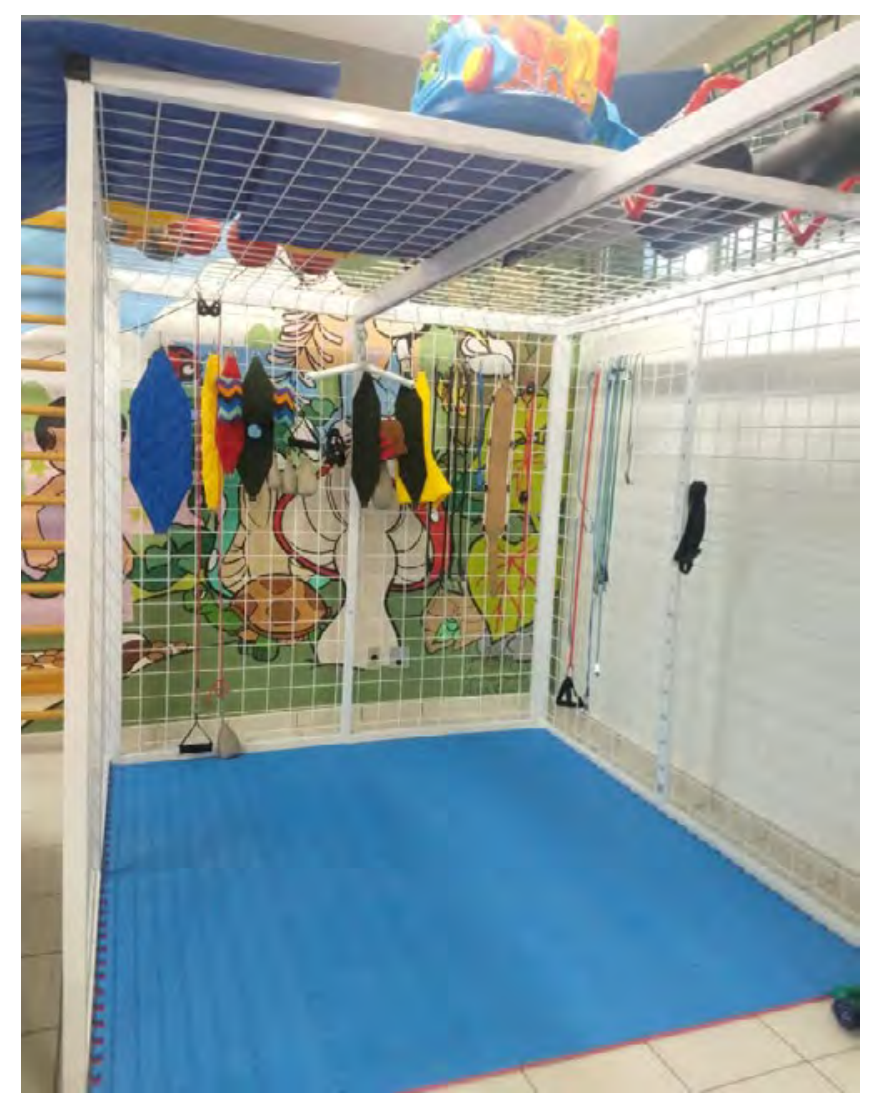

FONTE: Imagem original dos autores.

Ressalta-se que a questão do espaço apropriado era uma das principais dificuldades enfrentadas para a implantação e execução do projeto ao longo do tempo, já que a intervenção infantil requer ludicidade e metragem adequada para comportar pais, crianças, alunos e colaboradores. Além disso, outro grande desafio estava na aquisição de materiais específicos e que pudessem ser utilizados nas terapias, como: brinquedos de estimulação, equipamentos especializados, vídeo game (para gameterapia) e materiais para fabricação de órteses. A aquisição desses materiais foi obtida por meio de fomento fornecido pela universidade em cada semestre de execução do projeto.

Ao longo dos sete anos de permanente atuação, o PROPED teve cerca de 20 colaboradores e mais de 60 alunos envolvidos nas atividades e funcionamento do programa - números esses que representam satisfação e valorização. Dentro das atividades e objetivos propostos pelo projeto, está o incentivo ao ensino e a pesquisa, além da reabilitação infantil como principal atividade extensionista.

As atividades de ensino e pesquisa foram destinadas exclusivamente aos discentes e colaboradores, através da elaboração de casos clínicos atendidos no projeto, capacitação dos discentes quanto aos principais instrumentos de avaliações existentes na pediatria (por exemplo: Alberta Motor Infant Scale (AIMS), Gross Motor Function Measure (GMFM), Gross Motor Function Classification System (GMFCS) e Teste de Triagem de Desenvolvimento - DENVER II), bem como a produção de trabalhos científicos.

Aintervenção fisioterapêutica, incluindo avaliação e reabilitação, gerou o atendimento de 257 crianças, das quais muitas receberam órteses e todas participaram de atividades com orientação aos familiares, pais e/ou cuidadores. Os atendimentos foram, em geral, realizados pelos discentes e supervisionados pelos fisioterapeutas colaboradores e coordenadores. As crianças atendidas no projeto foram encaminhadas ao PROPED via unidades de saúde da cidade de Manaus/AM, como centros de reabilitação e maternidades que contam com programas de follow up, em que os pediatras e fisioterapeutas do serviço (alguns parceiros do projeto), triaram as crianças com atraso de desenvolvimento motor e as encaminharam para o serviço de intervenção precoce do programa. A tabela 1 mostra o perfil clínico, de acordo com o diagnóstico, das crianças atendidas no PROPED em percentuais. Tal perfil segue em consonância aos diagnósticos mais reportados na área da pediatria, incluindo a Paralisia Cerebral (PC) ou risco de PC (quando o diagnóstico ainda não está consolidado) e a Síndrome de Down, em congruência com os achados do estudo de Caricchio (2017), o qual avaliou a terapia com recursos lúdicos nas patologias neurológicas mais comuns da infância. 
Tabela 1 - Dados quantitativos referentes ao perfil clínico dos pacientes atendidos no PROPED atualmente

\begin{tabular}{lc} 
PATOLOGIAS & PACIENTES (\%) \\
Paralisia Cerebral (PC) ou Risco de PC & 30,4 \\
\hline Microcefalia & 13 \\
\hline Síndrome de Down & 8,7 \\
\hline Hidrocefalia & 8,7 \\
\hline Distrofia Muscular de Duchenne & 8,7 \\
\hline Miopatia Congênita & 8,7 \\
\hline Disfunções ortopédicas & 8,7 \\
\hline Mielomeningocele & 4,3 \\
\hline Paralisia Braquial Obstétrica & 4,3 \\
\hline TOTAL & 100 \\
\hline
\end{tabular}

FONTE: Dados desta pesquisa.

De modo geral, o projeto conseguiu promover programas de reabilitação infantil, direcionados para cada paciente e quadro patológico/deficiência, visando sempre a melhoria das condições físicas, funcionalidade, alcance dos marcos motores e melhora da qualidade de vida. Sem esquecer das orientações dadas aos familiares e/ou cuidadores, os quais possuem um importante papel na terapia da criança, pois, quando presentes, os fazem parte integrante nas atividades do dia a dia, levando à redução no estresse e ansiedade, favorecendo o desempenho das habilidades funcionais e o aumento do nível de independência das crianças. Além disso, a assistência e orientações fornecidas aos pais e cuidadores estão inseridas no conceito de "Prática Centrada na Família", o que promove inclusão familiar e reconhece a família como principal provedor de suporte e cuidado às crianças.

Outras ações envolvidas no PROPED estão relacionadas a identificação das necessidades específicas e multifatoriais de cada criança, as quais extrapolaram a competência da fisioterapia e exigiram encaminhamentos para outros serviços especializados de saúde, como: fonoterapia, terapia ocupacional, neuropsicoterapia, ortopedistas, neurologistas, nutricionistas, entre outros. Tais encaminhamentos geraram comunicação formal (por meio de documento específico de encaminhamento) e informal (por meio de contatos telefônicos ou encontros presenciais) que proporcionaram a discussão de casos clínicos voltados à assistência e suporte multidisciplinar à criança.

Desse modo, as ações extensionistas do PROPED tem cooperado com o desenvolvimento neuropsicomotor e social do público pediátrico, fornecendo não só atendimento de qualidade, mas também garantindo informações sobre as patologias/condições de saúde envolvidas, orientações sobre o prognóstico e tratamento e encaminhamentos para outras especialidades. Em adição, tal vivência forneceu aos discentes envolvidos no projeto, experiências valiosíssimas, uma vez que os mesmos, além de receberem treinamento especializado, puderam atuar como profissionais (supervisionados) e, assim, realizaram práticas clínicas efetivas, estabelecendo objetivos e condutas de tratamento, atuando na elaboração de cartilhas de orientação, realizando avaliações e aplicação de escalas confiáveis e validadas e, principalmente, enfrentando o desafio da experiência pediátrica, em que há a exigência de atuação lúdica atrelada aos complexos conceitos, técnicas, métodos e protocolos fisioterapêuticos.

\section{REFERÊNCIAS}

PEREIRA, Keila Ruttnig Guidony; SACCANI, Raquel; VALENTINI, Nadia Cristina. Cognição e ambiente são preditores do desenvolvimento motor de bebês ao longo do tempo. Fisioterapia e Pesquisa, São Paulo, v.23, n. 1, p. 59-67, 2016.

PEREIRA, Keila RG; VALENTINI, Nadia C.; SACCANI, Raquel. Brazilian infant motor and cognitive development: Longitudinal influence of risk factors. Pediatrics International, v.58, n.12, p. 1297-1306, 2016. 
BATAGLION, Giandra Anceski; MARINHO, Alcyane. Familiares de crianças com deficiência: percepções sobre atividade lúdica na reabilitação. Ciência \& Saúde Coletiva, Rio de Janeiro, v.21, n.10, p. 3101-3110, 2016.

SILVA, Allan dos Santos da; VALENCIANO, Paola Janeiro; FUJISAWA, Dirce Shizuko. Atividade lúdica na fisioterapia em pediatria: revisão de literatura. Revista Brasileira de Educação Especial, Marília, v.23, n.4, p.623-636, 2017.

BAENA, Cristina Pellegrino. Fisioterapia e integralidade: novos conceitos, novas práticas. Estamos prontos? Fisioterapia Brasil, Pretrolina v.12, n.2, 2011.

CARICCHIO, Milena Braga Maia. Tratar brincando: o lúdico como recurso da fisioterapia pediátrica no Brasil. Revista Eletrônica Atualiza Saúde, Salvador, v. 6, n. 6, p. 43-57, 2017.

RAVI, D. K.; KUMAR, Neelesh; SINGHI, P. Effectiveness of virtual reality rehabilitation for children and adolescents with cerebral palsy: an updated evidence-based systematic review. Physiotherapy, v.103, n. 3, p.245-258, 2017.

PINHEIRO, Paula Cassia Pinto de Melo et al. Therasuit e Pediasuit em crianças com paralisia cerebral. Revista Referências em Saúde, Goiânia, v.02 n.2, p.102-110, 2019. 